Editorial

\title{
Free Radicals and Antioxidants is now published with Elsevier
}

\section{Dear Readers}

It gives me great pleasure to announce that Free Radicals and Antioxidants (FRA) is now being published with Elsevier. With this collaboration we have taken an important step to further improve the quality of articles being published by FRA. We will continue to strive towards rapid review of submitted articles with emphasis on publishing articles with immediate translational relevance. The collaboration will also widen the scope of exposure for articles joining the Elsevier group of over 2500 journals. We are also working towards widening the scope of indexing for FRA articles in various databases including PubMed, SCOPUS and $\mathrm{SCI}$ etc.

One of FRAs goals has been to encourage new and upcoming researchers to share their scientific work with the rest of the community. We also encourage clinicians who have articles pertinent to the clinical use of antioxidants and the pathophysiological impact of drugs and diseases that modulate the level of free radicals in the body. We welcome original research, reviews, case reports and images. We also welcome interesting original images for inclusion on the cover page of the journal. Our Editorial Board comprises both basic researchers and clinicians and provides valuable input towards quality improvement of published articles. The online submission system provides an easy portal for authors to log in submit their work and follow its progress.

We encourage authors to contact us with any queries. We thank our reviewers for their time and invaluable service. We also acknowledge our Editorial Board members for their support. Finally, we wish to thank the authors for submitting such interesting original works to the journal. We are always excited to review your work and look forward to hearing from you.

Warm regards

Subhankar Chakraborty Editor, Free Radicals and Antioxidants http://www.frajournal.org

Available online $\mathrm{xxx}$ 